LBNL 62150

\title{
A simplistic view of hadron calorimetry
}

\author{
Donald E. Groom \\ 50R6008, Lawrence Berkeley National Laboratory, Berkeley CA 94720 \\ deg@lbl.gov
}

\begin{abstract}
All too often we rely on Monte Carlo simulations without worrying too much about basic physics. It is possible to start with a very simple calorimeter (a big cylinder) and learn the functional form of $\pi / e$ by an induction argument. Monte Carlo simulations provide sanity checks and constants. A power-law functional form describes test beam results surprisingly well. The prediction that calorimeters respond differently to protons and pions of the same energy was unexpected. The effect was later demonstrated by the CMS forward calorimeter group, using the most noncompensating calorimeter ever built. Calorimeter resolution is dominated by fluctuations in $\pi^{0}$ production and the energy deposit by neutrons. The DREAM collaboration has recently used a dual readout calorimeter to eliminate the first of these. Ultimate resolution depends on measuring neutrons on an event-by-event basis as well.
\end{abstract}

Keywords: Hadron calorimetry, hadron cascades, sampling calorimetry

PACS: $02.70 . \mathrm{Uu}, 29.40 . \mathrm{Ka}, 29.40 . \mathrm{Mc}, 29.40 \mathrm{Vj}, 34.50 . \mathrm{Bw}$

\section{Introduction}

After more than three decades of creative thought, development, and testing, hadron calorimeters have become highly evolved and sophisticated backbones of high-energy physics detectors. Their design concept remains the domain of experienced physicists who often rely on modern hadronic cascade simulation codes, but the actual design and construction has moved to expert engineering design teams. It is easy to lose sight of the underlying simplicity of the processes taking place inside the calorimeter. The object of this paper (colloquium) is to shed considerable baggage and to go back to the elemental physics situation.

The "calorimeter" we consider is nothing more than a cylinder which is long enough and big enough to completely contain the cascade produced by a, usually a pion or proton, arriving along the axis. It can be homogenous or sampling; if the latter, the sampling is sufficiently fine that the material can be regarded as uniform. There is no front em compartment and no rear catcher; these can be added after the conceptual framework is in place.

I do rely on Monte Carlo calculations made by other people-for the most part, the creators of HETC (CALOR), MARS, and FLUKA. The only thing of interest here is the $\pi^{0}$ fraction; this is a robust feature of all of the codes and has changed little over a decade or two of code improvement.

The objects are to understand the energy dependence of the $\pi / e$ ratio, the difference between pion and proton induced cascades, and, finally, the exciting new results from a dual-readout calorimeter. The content of this paper (colloquium) derives mainly from the 1994 paper by Gabriel et al.[1] (Paper I) and a paper presently in process with Nucl. 


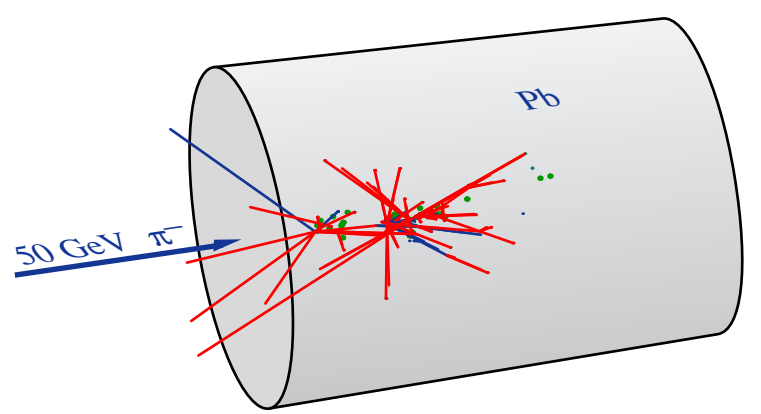

FIGURE 1. The "calorimeter" used in this discussion. The cylinder (usually lead or iron in the simulations) has a big enough radius and length to totally contain the hadronic cascade except for front-surface albedo losses, and the projectile, usually a pion but sometimes a proton, is incident along the axis.

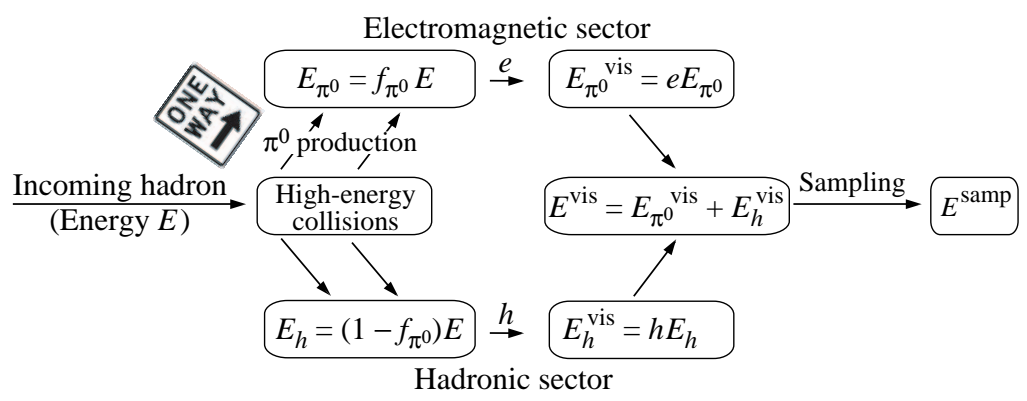

FIGURE 2. Energy flow in a hadronic cascade. A fraction $f_{\pi^{0}}$ (with energy-dependent mean $f_{\pi^{0}}^{0}$ ) is transferred to the electromagnetic sector through $\pi^{0}$ production in repeated hadronic inelastic collisions. The $\pi^{0}$ and hadronic energy deposits after the division are separately stochastic, and so must be treated as parallel stastical processes. Each produces a potentially detectable signal, whose sum $E^{\mathrm{vis}}$ is sampled.

Instrum. and Meth.[2] (Paper II).

\section{Electron and hadron energy deposit}

My model of energy fbw in a hadronic cascade is shown in Fig. 2. A hadron with energy $E$ generates a cascade in which there are repeated hadronic collisions. In each of these $\pi^{0}$ 's are produced, which immediately decay to photons. A fraction $f_{\pi^{0}}$ (with mean $f_{\pi^{0}}^{0}{ }^{1}$ ) of the energy is irrevocably removed from the hadronic part of the cascade in this way, and is instead deposited in electromagnetic (em) showers. It is converted to a potentially observable signal with efficiency $e$, which is in general different, usually larger, that the hadronic detection efficiency $h$. Gammas from nuclear excitation are considered as part of the hadronic signal, since the energy they carry scales as the hadronic fraction.

1 A superscript 0 is used to denote the mean of a stochastic variable. 

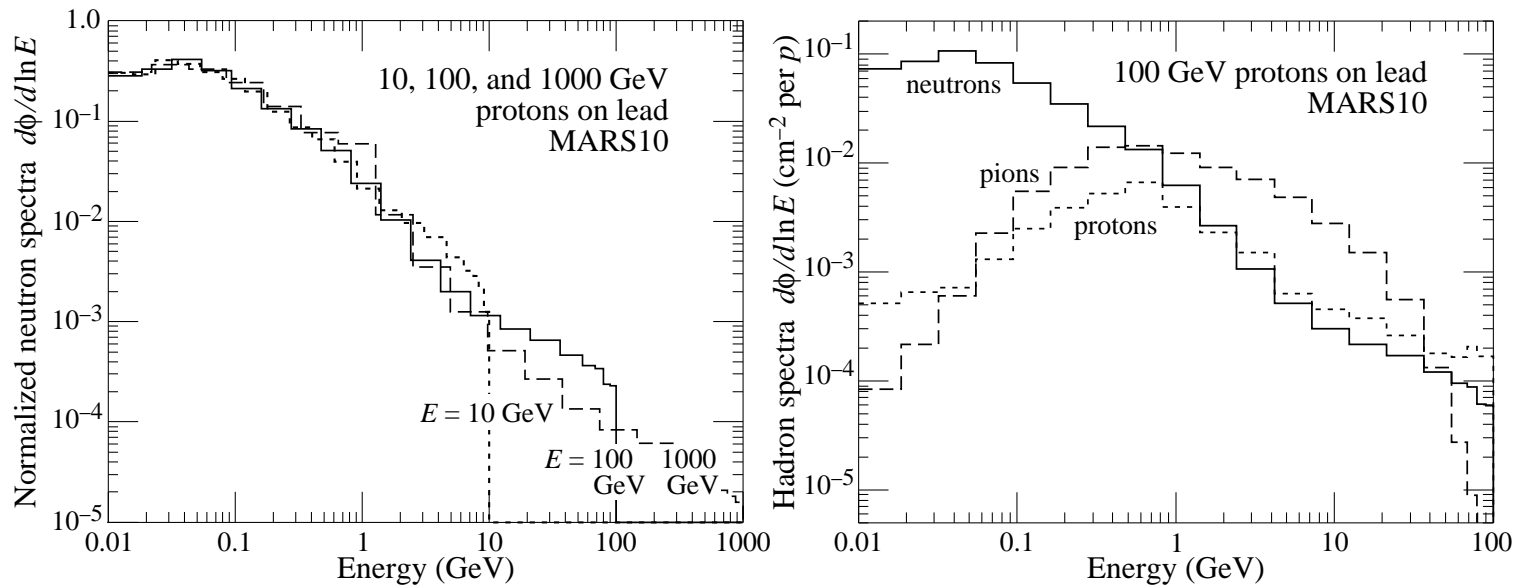

FIGURE 3. (a) MARS10 simulations of the neutron spectra in a lead beam stop for incident proton energies of 10, 100, and $1000 \mathrm{GeV}$. They are normalized for relative agreement at low energies to emphasize the shape similarity below the beam energy cutoff. (b) MARS10 simulation of the neutron, proton, and pion spectra for $100 \mathrm{GeV}$ protons on lead. At low energies the charged particles are removed by ionization loss. Above about $1 \mathrm{GeV}$ protons and neutrons have similar spectra. The relative numbers depend upon the material.

\section{The "universal spectrum"}

In either case, the cascade or shower grows exponentially until the lowest-energy particles stop or are absorbed. Except in the case of Cherenkov light, the ionization by charged particles in the sensitive part of the calorimeter constitutes the observable signal. In the em case much of the ionization is by electrons below the critical energy, of order $10 \mathrm{MeV}$ (13.8 MeV in $\mathrm{Fe}$ and $6.0 \mathrm{MeV}$ in $\mathrm{U}$ ). In the case of hadronic deposit, it is by ionization losses by pions, protons, neutron interaction products, Compton recoil electrons, spallation fragments, etc. The low-energy particles responsible for most of the deposit have some interesting features:

- The spectra have no memory of the initial hadron or even of the early stages of the cascade. A proton, pion, or kaon generates the same spectrum of a given species and the same ratios of the spectra of different species. For example, the relative low-energy particle spectra are the same for an incident pion or proton.

- There is also no memory of the energy of the incident hadron.

MAR10 simulations illustrate these points[1]. The neutron spectra shown in Fig. 3(a) are normalized for relative agreement. Over two orders of magnitude in the incident proton energy, the shapes of the neutron spectra below the lowest incident proton energy (or slightly lower) agree. The flux is 700 times higher at the peak than at $10 \mathrm{GeV}$; contributions to the total flux by high-energy particles have little affect. The neutron, pion, and proton fluxes shown in Fig. 3(b) have ratios dependent on the calorimeter environment but independent of the nature of the incident hadron.

The universality of the low-energy spectra in a given calorimeter environment has important consequences for calorimetry, among these the possibility of defining a constant 


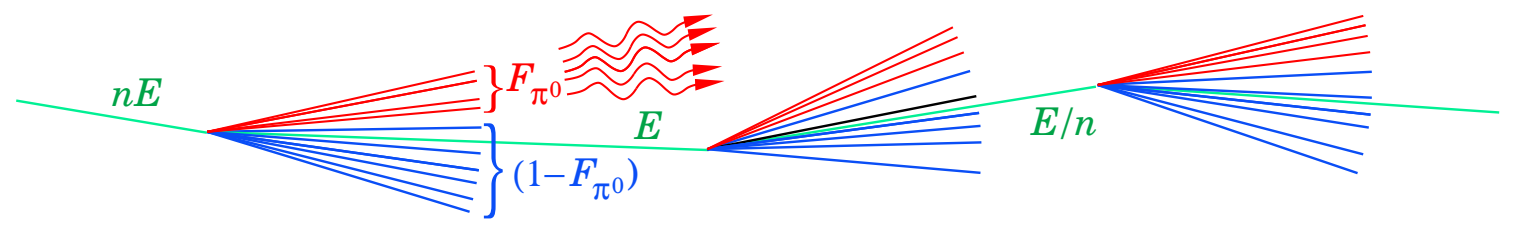

FIGURE 4. Cartoon of a hadronic cascade. It is assumed that in each generation the average energy of cascade particles decreases by a factor $n$ and than an average fraction $F_{\pi^{0}}$ of the energy leaves the hadronic sector via $\pi^{0}$ production.

hadron detection efficiency $h$.

\section{Can we understand the $\pi / e$ ratio from basic principles?}

\section{The mean hadronic fraction in a cascade}

Real hadronic cascades are lumpy affairs, often with a few separated regions of high energy deposit. A low-energy cascade of this sort is shown in Fig. 1. Dramatic examples of this were shown by Richard Wigmans in his talk at this Symposium. But in the spirit of my approach, we instead imagine the cartoon cascade shown in Fig. 4.

In this Figure, a hadron with energy $n E$ interacts with the production of $\pi^{0}$ 's and secondary hadrons. If the multiplicity is $n$, then an average hadron after the interaction has energy $E$. In this single collision an average energy fraction of $F_{\pi^{0}}$ goes into $\pi^{0}$ 's. (This is not to be confused with $f_{\pi^{0}}$, indicated in Fig. 2 as the total fraction going into $\pi^{0}$ 's in the cascade.)

Now let $A(E)$ be some measure of the level of hadronic activity induced by a hadron with energy $E$. There are many candidates: radioactivation, the length of all tracks made by particles with energies above some cutoff, the number of stars with energies above some cutoff, ionization energy deposit, the total energy of nuclear gamma rays, or, in the case of interest here, hadronic activity. So we can write

$$
A(n E)=\sum_{\text {secondaries } \neq \pi^{0}} A\left(E_{i}\right)
$$

since except for the ionization of a stray track or two the activity induced by $A(n E)$ has to be the same as that induced by the hadronic daughters.

So far this is incontestable; nothing has been said about the energy distribution of the 2nd generation hadrons. But now I replace the summation by $n$ times the activity induced by the average hadron. A fraction $F_{\pi^{0}}$ has been carried out of the hadronic sector by $\pi^{0}$ 's, so

$$
A(n E) \approx\left(1-F_{\pi^{0}}\right) n A(E) .
$$

This is immediately recognizable as the recursion equation for a power law, so we can write

$$
A(E)=K E^{m}
$$


Substitution into Eqn. 2 yields

$$
1-m=\frac{\ln \left(1 /\left(1-F_{\pi^{0}}\right)\right)}{\ln n}
$$

From an isotopic spin argument we might expect $F_{\pi^{0}}$ to be about $1 / 3$; the Monte Carlo's indicate that it is closer to $1 / 4$. The multiplicity $n$ might be 6 or 7 ; it has a $\ln E$ energy dependence but since we take the log again, the denominator doesn't vary much with energy. These rather hand-waving arguments say that $m$ is in the range $0.82-0.87$. It is ultimately an experimental number. From the construction, we must not expect the power-law approximation to work very well below about $10 \mathrm{GeV}$, although it seems to work down to about $5 \mathrm{GeV}$. It is asymptotically zero; at very high energies the cascade is nearly entirely electromagnetic.

If we say that $A(E)$ is the mean hadronic fraction $f_{h}^{0}$, then

$$
f_{h}^{0} \approx\left(E / E_{0}\right)^{m-1}
$$

Here $E_{0}$ is introduced as the scale factor for dimensional reasons, but it can be understood as roughly the threshold for $\pi$ - $p$ inelastic collisions, or about $1 \mathrm{GeV}$.

\section{The $\pi$ / e response ratio}

A calorimeter usually has a linear response $e E$ to electons, where $e$ is the efficiency for converting the em energy deposit to a visible signal. In a hadronic cascade a mean fraction $f_{\pi^{0}}^{0}$ is observed with efficiency $e$, and a mean fraction $f_{h}^{0}\left(=1-f_{\pi^{0}}^{0}\right)$ with efficiency $h$, so that the mean signal is $E\left(e f_{\pi^{0}}^{0}+h f_{h}^{0}\right)$. After a little manipulation, the ratio of pion to electron response is found to be

$$
\begin{array}{rcc}
\pi / e & = & 1-(1-h / e) f_{h}^{0} \\
& \approx & 1-(1-h / e)\left(E / E_{0}\right)^{m-1} \equiv 1-a E^{m-1} .
\end{array}
$$

In the second line we have explicitly used the power-law dependence given in Eqn. 5. It is important to note that only $a=(1-h / e) / E_{0}^{m-1}$ can be determined by measuring the energy dependence of $\pi / e$. Any claim about the value of $h / e$ rests on an assumed value for $E_{0}$. But since $E_{0}$ is raised to a small power, $a$ is insensitive to its exact value, and $E_{0} \approx 1 \mathrm{GeV}$ is often assumed.

Fits to a wide variety of test-beam data have been made. A subset is shown in Fig. 5.

\section{Inclusion of nuclear gamma rays}

Substantial em energy can be deposited by nuclear gamma rays. Most of this energy comes from slow neutron capture followed by nuclear deexcitation, which occurs on a time scale of $\mu$ s rather than ns. A typical acceptance gate is open for $100 \mathrm{~ns}$, and so some of the energy contributes to the visible signal. It is convenient to define this contribution 


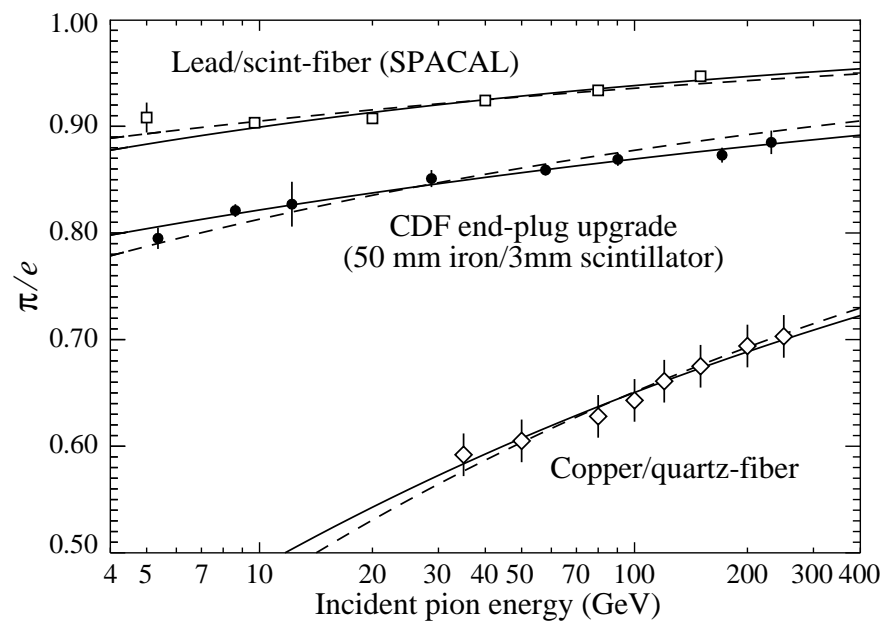

FIGURE 5. Fits to test-beam results for a lead/scintillator-fiber)[4], for the CDF upgrade endcap hadron calorimeter (50 $\mathrm{mm}$ iron/3 $\mathrm{mm}$ scintillator sheets)[5] and for a copper/quartz-fiber test calorimeter[6].

as $f_{h} f_{\gamma}$, so that the total em energy contribution to the visible signal is $E\left(f_{\pi^{0}}+f_{h} f_{\gamma}\right)$, with mean $E\left(f_{\pi^{0}}^{0}+f_{h}^{0} f_{\gamma}\right)$. We can repeat the algebra leading to Eqn. 6 to find

$$
\begin{array}{rcc}
\pi / e & = & 1-\left(1-h^{\prime} / e\right)\left(1-f_{\gamma}\right) f_{h}^{0} \\
& \approx & 1-\left(1-h^{\prime} / e\right)\left(1-f_{\gamma}\right)\left(E / E_{0}\right)^{m-1} \equiv 1-a E^{m-1} .
\end{array}
$$

The most important conclusion from Eqn. 7 is that the power law is retained, with the same experimental parameter $a$, even though part of the em contribution tracks with $f_{h}$. One can use either Eqn. 6 or Eqn. 7. The only difference is that the hadron detection efficiency $h^{\prime}$ does not contain contributions from nuclear gamma rays.

\section{The $p / \pi$ response ratio}

In a hadronic collision a leading particle (highest energy secondary) tends to have the same quark number as the incident particle. If it is a pion, there is a high probability that the leading particle is a $\pi^{0}$. If this is the case, a large fraction of the incident energy is removed to the electromagnetic sector; if not, a leading $\pi^{ \pm}$can dump a significant fraction of the energy into the em sector on the next collision. A smaller energy fraction (with mean $f_{\pi^{-}}^{0}$ ) is available for the hadronic sector than would be the case without leading particle effects. If the incident hadron is a proton, the leading particle is usually a proton or neutron, and the net effect is a larger mean hadronic fraction than in the pion case: $f_{p}^{0}>f_{\pi^{-}}^{0}$.

The "universal spectrum" idea tells us that the cascade's low-energy composition is the same in either case, not only as a function of energy but for either projectile. The ratio $f_{p}^{0} / f_{\pi^{-}}^{0}$ should therefore be the same at any incident energy, even though both $f_{\pi^{-}}^{0}$ and $f_{p}^{0}$ are functions of energy. 


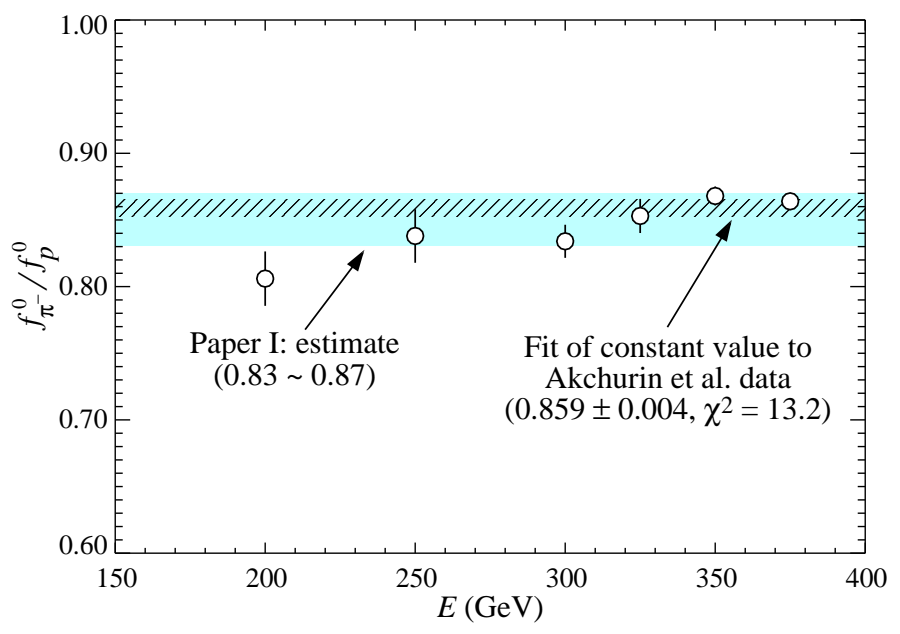

FIGURE 6. The mean hadronic fraction ratio $f_{\pi^{-}}^{0} / f_{p}^{0}$ as calculated from the copper/quartz-fiber calorimeter data of Ref. [3]. The gray band is the range expected from Paper I; the crosshatched band is a constant value fitted to the data. The PDG scale factor is 1.6.

But since they are different at a given energy, there should be a measurable $\pi / p$ ratio different from unity in a noncompensating calorimeter. We can rewrite Eqn. 6 for the incident pion and incident proton cases, and rearrange to get

$$
f_{\pi^{-}}^{0} / f_{p}^{0}=\frac{1-\pi / e}{1-p / e} \approx\left(E_{0 \pi^{-}} / E_{0 p}\right)^{1-m} .
$$

The factor $(1-h / e)$ cancels in the reduction. If the power-law approximation is invoked, as in the second line of Eqn. 8, the ratio is just the ratio of the scale energies to the $m-1$ power. It is clear from the equation that the statistical sensitivity is maximal for small $h / e$, when $\pi / e$ and $p / e$ are as small as possible. The opportunity to make the measurement was provided by the CMS quartz-fiber forward calorimeter test module (QFCAL), whose Cherenkov readout had very low sensitivity to hadrons. It had the lowest $h / e$ of any known calorimeter, about $1 / 5$. A plot of $f_{\pi^{-}}^{0} / f_{p}^{0}$ as a function of energy, derived from results presented in the paper, is shown in Fig. 6[3]. Since the constancy of the ratio follows from simple physical arguments, the apparent energy dependence in the Figure is not understood.

\section{Dual readout calorimeters}

The resolution of hadron calorimeters is worse that that of em calorimeters, mostly because of fluctuations in the $\pi^{0}$ content (in the case of noncompensating calorimeters) and fluctuations in neutron content. (Many of the neutrons which are produced endothermically do not "return" their energy before the acceptance gate of the calorimeter has closed.) The "Holy Grail" in the design of hadron calorimeters is to measure, correct for, and thus remove the effects of both of these fluctuations[7]. There have been many proposals concerning how to do this, but the most significant advance has been by the 


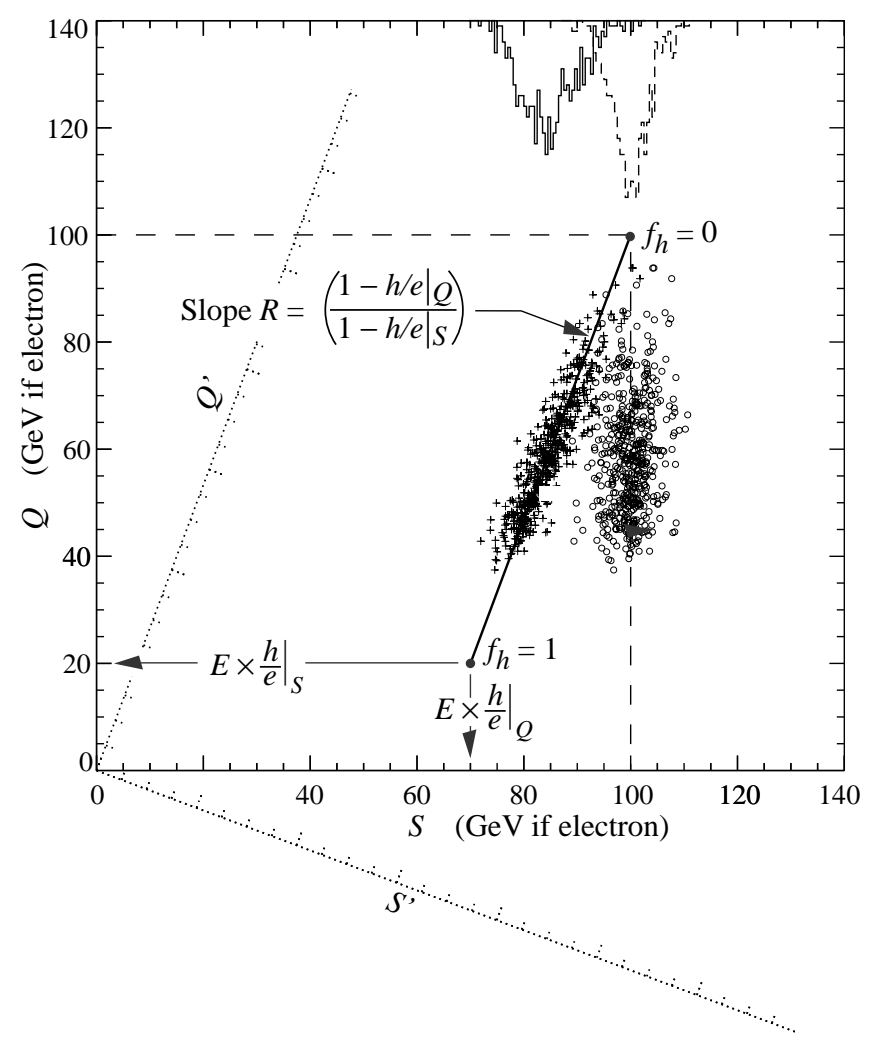

FIGURE 7. A toy model showing energy correction for $100 \mathrm{GeV}$ pions in an idealized DREAM calorimeter, where $Q$ is the response in the quartz-fiber readout and $S$ is the response in the scintillatorfiber readout. The observed "events" are shown by the +'s, and the corrected events by the o's. Rotating to a frame in which the $Q^{\prime}$ axis is parallel to the event locus provides an equivalent reduction.

DREAM collaboration[8]. Their test calorimeter consisted of copper tubes stuffed with 7 fibers each -3 of scintillator, and 4 of either quartz or acrylic nonscintillating fibers. Each kind had its own readout. The scintillators were sensitive to both photons and hadrons, while the nonscintillating fibers were sensitive only to Cherenkov light generated mostly by electrons. The "compensation contrast" was as large as possible, with $h / e \approx 0.7$ for the scintillators and $h / e \approx 0.2$ for the Cherenkov radiators. The Cherenkov output $(Q)$ vs the scintillator output $(S)$ is shown by the +'s in Fig. 6 for a set of $100 \mathrm{GeV}$ toy events. They scatter around the diagonal locus in the figure, whose position depends upon $h / e$ in each channel. The marginal distribution in $S$ is shown by the solid histogram at the top, with $\sigma=5.3 \%$. Given the locus (either by a fit to the data or by measuring the energy dependence of $\pi / e$ for each channel), the energy can be corrected for em content. With the definition $R=\left(1-h /\left.e\right|_{Q}\right) /\left(1-h /\left.e\right|_{S}\right)$, the corrected energy is

$$
E_{\mathrm{corr}}=\frac{R S-Q}{R-1}
$$

The circles in Fig. 7 show the same events after reduction via Eq. (9), and the dashed histogram shows the marginal distribution. The mean is $100.1 \mathrm{GeV}$, the fractional stan- 
dard deviation is $3.4 \%$, and there is no evident skewness. Complete compensation has been achieved by using the simultaneous readouts.

An alternative approach is to move to a rotated coordinate system $\left(S^{\prime}, Q^{\prime}\right)$ where the event locus is perpendicular to the $S^{\prime}$ axis. The marginal distribution on the $S^{\prime}$ axis has minimal width. After correction for foreshortening effects, this procedure yields a result which is algebraically identical to Eqn. 9.

\section{The future}

The remaining problem is to correct for fluctuations in the number of neutrons in each event. Ways to do this are under active investigation, particularly by the NLC "Fourth concept" group[9]. Neutron signatures include the lateral distribution of the energy deposition, ionization produced by $n-p$ elastically scattered protons in hydrogenous scintillator, and the highly ionizing spallation products. The possibility of observing the fast, blue, directional, polarized Cherenkov light in an inorganic scintillator is the object of present test beam work, using the scintillator $\mathrm{PbWO}_{4}[10]$. (The slow component has only a $50 \mathrm{~ns}$ decay time, $\lambda_{\max }=560 \mathrm{~nm}$ (yellow), but the scintillation efficiency is only $0.1 \%$ that of $\mathrm{NaI}$.) This scheme might achieve the em separation of DREAM with a single detector. It remains to do something like sandwiching $\mathrm{PbWO}_{4}$ with thin organic scintillator sheets to resolve the neutron component via $n$ - $p$ elastically scattered protons. For each event the $\mathrm{PbWO}_{4}$ scintillation signal, the $\mathrm{PbWO}_{4}$ Cherenkov signal, and the organic scintillator signal would be represented as a point in a data cube analogous to the two-dimensional "data square" shown in Fig. 7. It will be interesting to see if a correction formula as simple as Eqn. 9 can be found.

These are very promising directions, and it seems likely that the hadron calorimeter of the future will achieve something close to the energy resolution of em calorimeters.

\section{Appendix: The uselessness of $d E / d x$}

"The expression $\mathrm{dE} / \mathrm{dx}$ should be abandoned; it is never relevant to the signals in a particleby-particle analysis."-Hans Bichsel[11]

Calorimeters are often calibrated with muon beams. Here, as in other situations, it is sometimes assumed that the Bethe-Bloch $d E / d x^{2}$ (including density-effect corrections) is being measured. One plots a histogram of the signals at a given muon energy, calculates the average, and reduces it to the "mip," the signal that would be produced at the minimum of the Bethe-Bloch $d E / d x$ function at $\beta \gamma \approx 3-4$. The measurement is typically based on several hundred events, so the average is biased by events in the Landau tail and background events. Sometimes the more stable peak of the distribution, the "most probable" energy deposit, is used instead. In either case, particularly the former, the result for high-energy muons must also be corrected for radiative effects.

2 This is the mean $d E / d x$; we follow convention by ignoring the fact that it is negative. 
To understand the situation, it is convenient to write[12]:

$$
\frac{d E}{d x}=\left(\begin{array}{l}
\text { Contribution from } \\
\text { low-energy electron } \\
\text { scattering }
\end{array}\right)+\left(\begin{array}{l}
\text { Contribution from } \\
\text { high-energy elec- } \\
\text { tron scattering, } \\
T_{\text {cut }}<T_{\max }
\end{array}\right)+\left(\begin{array}{l}
\text { Contribution } \\
\text { from } \delta \text { rays, } \\
T_{\text {cut }} \leq T<T_{\max }
\end{array}\right)
$$

$d E / d x$ is derived (nearly) this way[13, 14], except that the last two terms are usually combined. (In his very thorough treatment, Fano[14] also introduces a third term in the region where the low-energy an high-energy terms meet.) The division into $d E / d x$ contributions from low-energy electron scattering and high-energy electron scattering is made on the basis of the approximations used. For example, in calculating the highenergy electron contribution, atomic binding energy can be neglected.

Semiclassically, the low-energy region corresponds to distant collisions in which the atom is excited or frees an electron with energy somewhat larger than its binding energy. We might imagine the incident particle's electric field as extending outward from the smallest impact parameter consistent with the upper energy cutoff. As the incident particle becomes more and more relativistic, the field fhttens and extends. This produces a $\ln \beta \gamma$ additive contribution. But this extension is limited by polarization of the medium, the "density effect." Sternheimer developed a rather accurate theory for predicting the effect in any material[15]. Asymptotically,

$$
\delta / 2 \rightarrow \ln \left({ }^{-} h \omega_{\beta} / I\right)+\ln \beta \gamma-1 / 2 \text {. }
$$

where the plasma energy ${ }^{-} h \omega_{\beta}$ and effective ionization potential $I$ are not of interest here. The important points are that (a) the density-effect term goes with the low-energy contribution, not elsewhere, and (b) this $\ln \beta \gamma$ cancels the $\ln \beta \gamma$ in the low-energy contribution, as we might expect on physical grounds. The first term's contribution to $d E / d x$ thus approaches a constant as the energy increases. Both of these points are widely misunderstood.

Together, the sum of the first two terms in Eqn. 10 is the "restricted energy loss"[16], which means that $d E / d x$ is calculated with the prohibition of single energy transfers in excess of some arbitrary $T_{\text {cut }} \leq T<T_{\max }$. $T_{\max }$ is the kinematic limit on kinetic energy transfer to one electron. The middle (second) term depends only on $\beta$ as well, and so also quickly approaches a constant. Examples of restricted energy loss are shown in Fig. 8.

It is easy to integrate $T d^{2} N / d x d T$ [16] over the interval $T_{\text {cut }}<T<T_{\max }$ to obtain the last term in Eqn. 10. Here $N$ is the number of $\delta$ rays. The result is exactly the difference between the full $d E / d x$ and the restricted $d E / d x$. The integral is proportional to $\ln T_{\max }$, which automatically introduces a $\ln \beta \gamma$ dependence. The asymptotic logarithmic rise of $d E / d x$ comes entirely from the $\delta$ rays. An integration to find $d N / d x$ shows that the number of $\delta$ rays above $T_{\text {cut }}$ in a reasonable detector is very small, even for $T_{\text {cut }}$ as low as the minimum energy loss in the detector. For example, a $500 \mathrm{MeV}$ pion traversing a $300 \mu \mathrm{m}$ thick silicon detector produces a $\delta$ ray with energy greater than "1 mip" in only one event out of 20. The increase in the number and length of $\delta$-ray tracks along the trajectory of a particle in a bubble chamber was once used to estimate the particle's energy. 


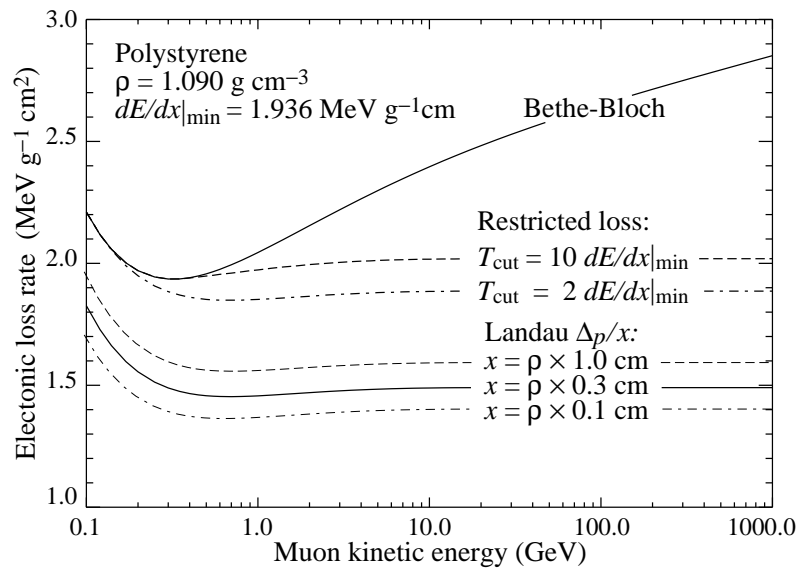

FIGURE 8. Bethe-Bloch $d E / d x$, two examples of restricted energy-loss rate, and the Landau most probable energy deposit per unit thickness in polystyrene scintillator, in which $d E /\left.d x\right|_{\min }=$ $1.936 \mathrm{MeV} \mathrm{g}^{-1} \mathrm{~cm}^{2}$. The incident particles are muons. The calculations of $\Delta_{p} / x$ were by Hans Bichsel.

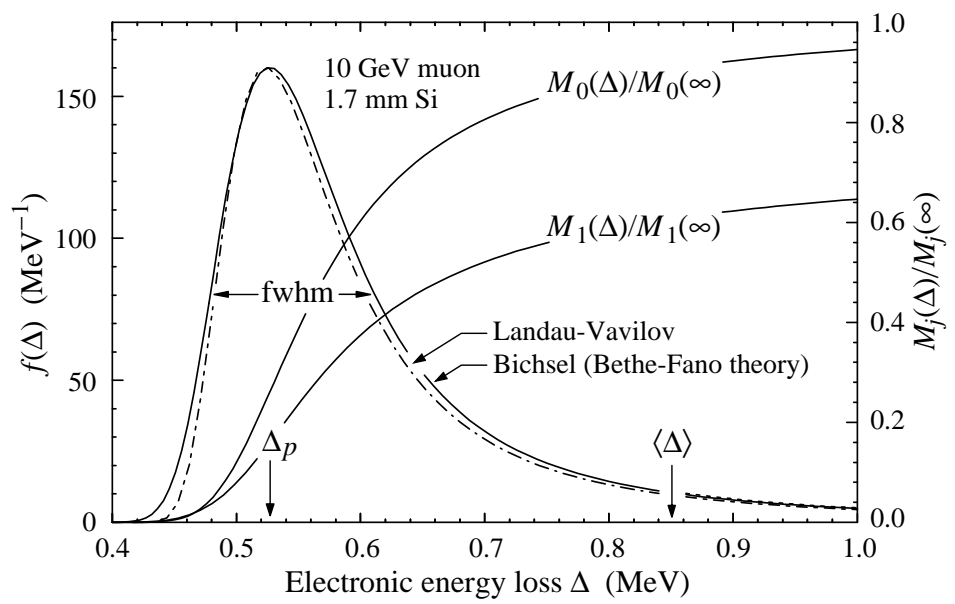

FIGURE 9. Bichsel's calculations of the electronic energy deposit distribution for a $10 \mathrm{GeV}$ muon traversing a $1.7 \mathrm{~mm}$ silicon detector (which has roughly the stopping power of a 3-mm thick scintillator) [11, 17, 18, 20]. The Landau-Vavilov function (dot-dashed) uses a Rutherford cross section without atomic binding corrections but with a maximum kinetic energy transfer limit $T_{\max }$. The solid curve was calculated using Bethe-Fano theory. $M_{0}(\Delta)$ and $M_{1}(\Delta)$ are the cumulative 0th and 1st moments of $f(\Delta)$, respectively. The fwhm of the Landau-Vavilov function is about $4 \xi$ for detectors of moderate thickness.

For detectors of moderate thickness $x$ (e.g., the scintillator tiles or LAr cells used in calorimeters $),{ }^{3}$ the energy-loss probability distribution $f(\Delta ; \beta \gamma, x)$ is adequately described by the Landau (or Landau-Vavilov-Bichsel) distribution[19, 20, 21]. A special

${ }^{3} G \lesssim 0.05-0.1$, where $G$ is given by Rossi [[13], Eq. 2.7.0]. It is Vavilov's $\kappa[19]$. 
case is shown in Fig. 9. The most probable energy loss is given by

$$
\begin{gathered}
\Delta_{p}=\xi\left[\ln \frac{2 m_{e} c^{2} \beta^{2} \gamma^{2}}{I}+\ln \frac{\xi}{I}+j-\beta^{2}-\delta(\beta \gamma)\right] \\
\underset{\beta \gamma \gtrsim 100}{\longrightarrow} \xi\left[\ln \frac{2 m_{e} c^{2} \xi}{(-h \varphi)^{2}}+j\right] .
\end{gathered}
$$

where $\xi=0.153537\langle Z / A\rangle\left(x / \beta^{2}\right) \mathrm{MeV}$ for a detector whose thickness $x$ is in $\mathrm{g} \mathrm{cm}^{-2}$, and $j=0.200 .{ }^{4}$ While $d E / d x$ is independent of thickness, $\Delta_{p} / x$ scales as $a \ln x+b$. The density correction $\delta(\beta \gamma)$ was not included in Landau's or Vavilov's work, but it was later included by Bichsel[20]. For scintillator (polystyrene), the mean excitation energy is $68.7 \mathrm{eV}$ and the plasma energy ${ }^{-} h \omega$, is $21.8 \mathrm{eV}$.

For the case shown in Fig. 9, the mean energy loss is about $50 \%$ greater than the most probable loss. With increasing energy it moves to the right because of occasional $\delta$-ray production. With $T_{\max }$ in the $\mathrm{GeV}$ region, the mean is strongly influenced by contributions to the tail hundreds or thousands of times the range of the plot. It makes sense to determine the most probable energy loss, not the ephemeral and undependable average.

Radiative contributions to $d E / d x$ rise almost linearly with energy, becoming as important as ionization losses at some "muon critical energy" $E_{\mu c}: 1183 \mathrm{GeV}$ in plastic scintillator, $347 \mathrm{GeV}$ in iron and $141 \mathrm{GeV}$ in lead[12, 23]. The contributions are significant well below the critical energy. Monte Carlo calculations by Striganov and collaborators[24] indicate that, while a high-energy shoulder appears on the energy-loss distribution, the most probable energy loss increases only slightly in "thin" absorbers, e.g., for $1000 \mathrm{GeV}$ muons incident on $100 \mathrm{~g} \mathrm{~cm}^{-2}$ of iron. They regard radiative effects as "important" when the most probable height of the normalized energy-loss distributions are lowered by $\gtrsim 10 \%$ when radiative effects are included. This can be the case under some circumstances.

\section{Acknowledgments}

I am indebted to a large fraction of my calorimetry and radiation physics friends for profitable discussions in the course of formulating these ideas, but particularly so to Richard Wigmans and my collaborators on Paper I: Tony Gabriel, P. K. Job, Nikolai Mokhov, and Graham Stevenson. Conversations with and input from Nural Akchurin, Alberto Fassó, Alfredo Farrari, and John Hauptman have been especially welcome and useful. The $d E / d x$ appendix is the result of long conversations with Hans Bichsel.

This work was supported by the U. S. Department of Energy under Contract No. DEAC02-05CH11231.

\footnotetext{
${ }^{4}$ Rossi[13], Talman[22], and others give somewhat different values for $j$. The most probable loss is not sensitive to its value.
} 


\section{REFERENCES}

1. T. A. Gabriel, D. E. Groom, P. K. Job, N. V. Mokhov, and G.R. Stevenson, Nucl. Insrum. and Meth. Phys. Res., Sect. A 338 (1994) 336-347.

2. D. E. Groom, Nucl. Insrum. and Meth. Phys. Res., Sect. A (in process) (2006); arXiv:physics/0605164 v3.

3. N. Akchurin et al., Nucl. Insrum. and Meth. Phys. Res., Sect. A 408 (1998) 380.

4. D. Acosta et al., Nucl. Insrum. and Meth. Phys. Res., Sect. A 308 (1991) 481.

5. J. B. Liu, "Testbeam results for the CDF endplug hadron calorimeter," Proc. VII Inter. Conf. on Calorimetry in High Energy Physics, Tucson, Arizona, 9-14 November 1997, ed. E. Cheu, T. Embry, J. Rutherfoord, R. Wigmans, World Scientific, (1998) 237-240.

6. N. Akchurin et al., Nucl. Insrum. and Meth. Phys. Res., Sect. A 399 (1997) 202.

7. R. Wigmans, "Quartz Fibers and the Prospects for Hadron Calorimetry at the 1\% Resolution Level," Proc. VII Inter. Conf. on Calorimetry in High Energy Physics, Tucson, Arizona, 9-14 November 1997, ed. E. Cheu, T. Embry, J. Rutherfoord, R. Wigmans, World Scientific, (1998) 182-193.

8. $\quad$ N. Akchurin et al., Nucl. Insrum. and Meth. Phys. Res., Sect. A 537 (2005) 537

9. P. Le Du et al., "Detector outline document for the Fourth Concept Detector at the International Linear Collider,"

http://physics.uoregon.edu/ lc/wwstudy/concepts / (May 2006).

10. R. Wigmans, private communication (2006).

11. H. Bichsel, Nuc. Insrum. and Meth. Phys. Res., Sect. A 562 (2006) 154-197.

12. D. E. Groom, N. V. Mokhov, and S. I. Striganov, Atomic and Nuclear Data Tables 78 (2001) 183.

13. B. Rossi, High-Energy Particles, (Prentice-Hall, Inc., Englewood Cliffs, NJ, 1952).

14. U. Fano, Ann. Rev. Nucl. Sci. 13 (1963) 1.

15. R. M. Sternheimer, Phys. Rev. 88, 851 (1952); Phys. Rev. 93, 351 (1953); Phys. Rev. 103, 511 (1956); Phys. Rev. 145, 247 (1966); Phys. Rev. 164, 349 (1967).

16. W.-M. Yao et al., "The Review of Particle Physics," J. Phys. G 33 (2006) 1.

17. H. Bichsel, private communications (2006).

18. H. Bichsel, Ch. 87 in the Atomic, Molecular and Optical Physics Handbook, G. W. F. Drake, editor (Am. Inst. Phys. Press, Woodbury NY, 1996).

19. P. V. Vavilov, Sov. J. Phys. JETP 5 (1957) 749.

20. H. Bichsel, Rev. Mod. Phys. 60 (1988) 663.

21. L. Landau, J. Phys. VIII, (1944) 201; P. V. Vavilov, Sov. J. Phys. JETP 5 (1957) 749.

22. R. Talman, Nucl. Insrum. and Meth. 159 (1979) 189.

23. http://pdg.lbl.gov/AtomicNuclearProperties

24. N. V. Mokhov, S I. Striganov, A. V. Uzunian, On Fluctuations of Energy Losses of Ultrarelativistic Muons, (in Russian), IFVE-80-56, (Serpukhov, IHEP) (Apr 1980), 12 pp. 